\title{
BROTAÇÃO E FERTILIDADE DE GEMAS EM UVAS SEM SEMENTES NO VALE DO SÃO FRANCISCO ${ }^{1}$
}

\author{
PATRÍCIA COELHO DE SOUZA LEÃO² \& EMANUEL ELDER GOMES DA SILVA
}

\begin{abstract}
RESUMO- Com o objetivo de avaliar a porcentagem de brotação e índice de fertilidade de gemas de cinco variedades de uvas sem sementes em cinco diferentes datas de poda, realizou-se um experimento no Campo Experimental de Bebedouro, da Embrapa Semi-Árido, em Petrolina-PE. O delineamento experimental foi em parcelas subdivididas, constituídas por cinco variedades de uvas sem sementes como tratamentos principais e cinco diferentes épocas de poda como tratamentos secundários, utilizando-se dez repetições constituídas por uma planta. As variedades utilizadas foram Perlette, Thompson Seedless, Marroo Seedless, Catalunha e Superior Seedless, e as épocas de poda foram: 18 e 19/12/2000; 18 a 21/06/2001; 23 a 29/10/2001; 29/01 a 05/02/2002 e 08 a 12/04/2002. As variáveis estudadas foram a porcentagem de brotação e o índice de fertilidade real das gemas determinado pela razão número de cachos por número de gemas. A fertilidade real de gemas foi analisada entre a $1^{\mathrm{a}}$ e $10^{\mathrm{a}}$ gema da vara. Os maiores valores médios para brotação e fertilidade foram observados nas variedades Marroo Seedless e Perlette, sendo que as podas realizadas nos meses de junho de 2001 e abril de 2002 favoreceram o aumento da brotação e da fertilidade das gemas na maioria das variedades.
\end{abstract}

Termos para indexação: videira, variedades, comportamento, frutificação

\section{BUDDING AND FRUITFULNESS OF SEEDLESS GRAPE IN THE SÃO FRANCISCO RIVER VALLEY}

ABSTRACT - Aiming to evaluate the bud burst percentage and fruitfulness index of five varieties of seedless grape in five different pruning dates, an experiment was carried out in the Experimental Station of Bebedouro, of Semi-arid Embrapa, in Petrolina-PE. The experimental design was in split plot, constituted by five varieties as main treatments, five different pruning times as secondary treatments and being used ten repetitions (plants). The varieties were Perlette, Thompson Seedless, Marroo Seedless, Catalunha and Superior Seedless, and the pruning times were the following ones: $12 / 18$ to $12 / 19 / 2000 ; 06 / 18$ to $06 / 21 / 2001 ; 10 / 23$ to $10 / 29 / 2001 ; 01 / 29$ to $02 / 05 / 2002$ and $04 / 08$ to $04 / 12 / 2002$. The studied variables were the percentage of budbreak and the fruitfulness index, calculated by the ratio number of bunches/number of buds. The fruitfulness was evaluated from the $1^{\text {st }}$ to the $10^{\text {th }}$ buds of the cane. The best results for budding and fruitfulness were obtained in the Marroo Seedless and Perlette varieties, and pruning in June of 2001 and April of 2002 increased sprouting and fruitfulness in most of the varieties.

Index terms: grapevine, variety, behaviour, fruitfulness

\section{INTRODUÇÃO}

A produção de uvas sem sementes tem apresentado rápida expansão no Submédio São Francisco, sendo a quase totalidade comercializada no mercado externo, onde tem alcançado preços até três vezes superiores que os da uva 'Itália'. A principal variedade é a Superior Seedless ou Festival, que se caracteriza por apresentar menor produtividade que as variedades com sementes cultivadas na região e ainda uma grande irregularidade de produção entre as safras.

$\mathrm{O}$ número de cachos constitui-se num dos principais componentes da produtividade e pode ser determinado pela poda e pela fertilidade das gemas. A fertilidade das gemas pode ser definida como a capacidade que apresentam para se diferenciar de vegetativas em frutíferas, podendo ser considerada como medida quantitativa do potencial de uma planta em produzir frutos. A diferenciação floral na videira e em outras plantas perenes ocorre durante a fase de crescimento vegetativo do ciclo anterior e envolve três estádios bem definidos: formação dos "anlage", formação dos primórdios de inflorescência e formação das flores (Srinivasan \& Mullins, 1981). A diferenciação das gemas tem início nas gemas basais e continua em direção a porção apical da brotação.

Essa característica depende de cada variedade, mas em uma mesma variedade pode sofrer grandes variações de um ciclo para outro, onde o clima pode exercer grande influência sobre a fertilidade de gemas.

A luminosidade diária, temperaturas acima de $30^{\circ} \mathrm{C}$ e luz solar incidente sobre as gemas são os principais fatores climáticos que atuam sobre o aumento da diferenciação floral (Baldwin, 1964; Buttrose, 1969; 1970; 1974; Rives, 2000; Sommer et al., 2000). Por outro lado, condições de manejo do vinhedo que podem afetar a temperatura e a luz solar incidente, tais como o sombreamento (May \& Antcliff, 1963), a direção de crescimento dos ramos (May, 1966), a desponta e a desbrota de ramos (Lavee et al., 1967), os sistemas de condução (Sommer et al.,
2000) e outros aspectos do manejo têm sido estudados por diversos autores.

O conhecimento da posição das gemas férteis para cada variedade é de fundamental importância na definição do tipo de poda a ser empregada no vinhedo. Podem ser encontradas referências de resultados obtidos para diferentes variedades, em regiões de produção muito distintas, como o Nordeste do Brasil (Leão \& Pereira, 2001), sul do Brasil (Tonietto \& Czermainski, 1993), Venezuela (Valor \& Bautista, 1997), México (Murrieda, 1986) e Itália (Sansavini \& Fanigliulo, 1998). Entretanto, existem poucas informações sobre o comportamento de variedades em condições de clima tropical, como aqueles predominantes no Submédio São Francisco. Esse conhecimento é um importante subsídio para se estabelecer técnicas mais racionais de poda que resultem no aumento de produtividade dos vinhedos.

O presente trabalho teve como objetivo avaliar a percentagem de brotação e índice de fertilidade de gemas de cinco variedades de uva sem sementes em cinco diferentes datas de poda nas condições do Submédio São Francisco.

\section{MATERIAL EMÉTODOS}

O experimento foi conduzido no Campo Experimental de Bebedouro da Embrapa Semi-Árido, em Petrolina - PE, 909'S , 40²2'O e altitude média de $365,5 \mathrm{~m}$.

Segundo Köeppen, o clima da região está classificado como tipo Bswh, sendo a temperatura média anual de $26,4^{\circ} \mathrm{C}$, com média das mínimas de $20,6^{\circ} \mathrm{C}$, e média das máximas de $31,7^{\circ} \mathrm{C}$. A Figura 2 apresenta médias mensais de temperatura, insolação e radiação durante o período de realização do experimento.

O delineamento experimental foi em parcelas subdivididas onde os tratamentos principais foram constituídos pelas variedades Perlette, Thompson Seedless, Marroo Seedless, Catalunha e Superior Seedless,

\footnotetext{
${ }^{1}$ (Trabalho 033/2003). Recebido: 27/02/2003. Aceito para publicação: 18/09/2003. Apoio financeiro Banco do Nordeste-FUNDECI.

${ }^{2}$ Eng $^{\mathrm{a}}$ Agrônoma, M.Sc. Pesquisadora Embrapa Semi-Árido, Caixa Postal 23, Zona Rural, Petrolina, PE, 56.300-970; Fone: (87)3862-1711; patricia@cpatsa.embrapa.br.

${ }^{3}$ Eng $^{\circ}$ Agrônomo, M.Sc. Bolsista CNPq; emanoel@cpatsa.embrapa.br.
} 
e os tratamentos secundários por cinco épocas de poda entre dezembro de 2000 à abril de 2002, a saber: 18 e 19/12/2000; 18 a 21/06/2001; 23 a 29/ 10/2001; 29/01 a 05/02/2002 e 08 a 12/04/2002.

As videiras enxertadas no porta-enxerto IAC 572 estavam com três anos de idade, conduzidas em latada no espaçamento 4,0 x 2,0 m, submetidas à irrigação localizada por gotejamento.

A poda utilizada foi do tipo mista com varas e esporões, mantendo-se varas com aproximadamente dez a doze gemas e esporões com duas a três gemas. Imediatamente após a poda, os ramos foram pulverizados com Dormex ${ }^{\circledR}$ em uma concentração de $5 \%$ do produto comercial para induzir e uniformizar a brotação das gemas.

A percentagem de brotação foi calculada pela relação entre o número de gemas brotadas e o número total de gemas; e o índice de fertilidade real foi obtido dividindo-se o número total de cachos pelo número total de gemas da planta.

Para se determinar a percentagem de gemas brotadas e o índice de fertilidade real para cada posição de gema, realizou-se uma avaliação aproximadamente entre o décimo e o trigésimo dia após a poda antes de se realizar a desbrota. Esta avaliação foi efetuada para todas as varas de produção e esporões, da $1^{\mathrm{a}}$ a $10^{\mathrm{a}}$ gema do ramo, em dez plantas ou repetições por variedade. Foram registrados o número total de gemas, número total de gemas brotadas, número total de cachos e número de cachos por posição de gema.

Os resultados foram analisados estatisticamente, utilizandose o programa SAS, estabelecendo-se a comparação das médias pelo teste de Duncan ao nível de $1 \%$ de probabilidade.

\section{RESULTADOS E DISCUSSÃO}

A análise de variância demonstrou serem significativos os efeitos de variedades e épocas de poda sobre a brotação e a fertilidade de gemas. A interação entre os efeitos de variedades e épocas de poda também foi significativa a $1 \%$ de probabilidade, indicando que o comportamento das variedades em relação à brotação e fertilidade de gemas pode variar segundo a época de poda (Tabelas 1 e 2), e em uma mesma época de poda, as cinco variedades apresentam diferenças significativas para brotação e fertilidade de gemas. Estes resultados comprovam que brotação, e sobretudo a fertilidade das gemas, são características que dependem não somente da variedade, mas que são altamente influenciadas pelas condições climáticas durante o período do ciclo em que ocorre a diferenciação das gemas, o que está de acordo com diversos autores (Baldwin, 1964; Buttrose, 1969; 1970; 1974; Rives, 2000; Sommer et al., 2000).

A brotação média variou de $62,42 \%$ na variedade Catalunha até $75,56 \%$ na 'Perlette'. A poda realizada entre 18 a 19 de dezembro de 2000 foi aquela onde foram observados os menores valores para brotação, diferindo significativamente dos demais tratamentos em todas as variedades. $\mathrm{O}$ valor médio para percentagem de brotação nesta época de poda foi de apenas $44,75 \%$. Por sua vez, a brotação das gemas foi mais elevada para as podas realizadas entre 18 a 21 de junho de 2001 e 8 a 12 de abril de 2002, não diferindo significativamente entre si nestas duas épocas de poda, à exceção da variedade Catalunha, onde obteve-se para a poda de fevereiro de 2002, o melhor resultado entre todos os ciclos, isto é, $84,78 \%$, diferindo significativamente das demais épocas.

Os maiores índices de fertilidade real de gemas podem ser observados, de acordo com a Tabela 2, na variedade Marroo Seedless, diferindo significativamente das demais variedades em todas as épocas de poda, com uma média geral de 0,37 , seguida pela 'Perlette' com 0,17. As variedades Thompson Seedless, Superior Seedless e Catalunha apresentaram fertilidade real muito baixa, não diferindo entre si nas cinco épocas de poda estudadas e apresentando uma média geral de respectivamente 0,$14 ; 0,12$ e 0,10 .

Em trabalho anterior realizado nesta região, obteve-se índices de fertilidade de gemas mais elevados para as variedades Marroo Seedless $(0,89)$ e Thompson Seedless $(0,68)$ (Leão \& Pereira, 2001), possivelmente em virtude das podas terem sido mais longas, analisando-se 15 gemas nas varas. Por outro lado, os resultados obtidos estão próximos daqueles observados por Camargo et al. (1996) nas variedades Catalunha $(0,18)$ e Perlette $(0,21)$.

A fertilidade de gemas nas cinco variedades, à exceção de 'Perlette', diferiu significativamente entre as diferentes épocas de poda. A poda realizada entre 8 a 12 de abril de 2002 foi a que promoveu a maior fertilidade de gemas, à exceção de 'Perlette', em todas as variedades. Outra época de poda favorável para a fertilidade das gemas foi em junho de 2001, à exceção da variedade Superior Seedless (Figura

TABELA 1 - Valores médios para percentagem (\%) de brotação e coeficiente de variação (C.V.) de cinco variedades de uvas sem sementes em diferentes épocas de poda, Petrolina, PE. ${ }^{1}$

\begin{tabular}{|c|c|c|c|c|c|c|}
\hline \multirow{2}{*}{$\begin{array}{c}\text { Épocas de poda } \\
\text { (mês/ano) }\end{array}$} & \multicolumn{6}{|c|}{ Variedades } \\
\hline & Perlette & Thompson Seedless & Marroo Seedless & Catalunha & Superior Seedless & Média \\
\hline $12 / 2000$ & $61,56 \quad \mathrm{bA}$ & $30,93 \mathrm{cC}$ & $50,20 \quad \mathrm{~dB}$ & $31,87 \quad \mathrm{dC}$ & $49,21 \quad \mathrm{cB}$ & 44,75 \\
\hline $06 / 2001$ & 75,63 bcA & 82,09 aAB & 94,11 aA & 66,98 & 75,63 abBC & 78,89 \\
\hline $10 / 2001$ & 75,34 aA & $59,77 \quad$ bB & $63,08 \quad \mathrm{cdAB}$ & 56,13 & 77,19 abA & 66,30 \\
\hline $02 / 2002$ & 79,16 aAB & 77,33 aAB & $69,98 \quad$ bcB & 84,78 aA & 68,77 & 76,00 \\
\hline $04 / 2002$ & 86,13 aA & 77,78 aAB & 82,45 abAB & 72,32 bB & 79,58 aAB & 79,65 \\
\hline Média & 75,56 & 65,58 & 71,96 & 62,42 & 70,08 & \\
\hline C.V. $(\%)$ & 14,00 & 10,76 & 20,22 & 13,82 & 8,22 & \\
\hline
\end{tabular}

${ }^{1}$ Médias seguidas pela mesma letra minúscula na coluna e maiúscula na linha não diferem entre si pelo teste de Duncan a $1 \%$ de probabilidade

TABELA 2 - Valores médios para índice de fertilidade real de gemas e coeficiente de variação (C.V.) de cinco variedades de uvas sem sementes em diferentes épocas de poda, Petrolina, PE. ${ }^{1}$

\begin{tabular}{|c|c|c|c|c|c|c|}
\hline \multirow{2}{*}{$\begin{array}{l}\text { Épocas de poda } \\
\text { (mês/ano) }\end{array}$} & \multicolumn{6}{|c|}{ Variedades } \\
\hline & Perlette & Thompson Seedless & Marroo Seedless & Catalunha & $\begin{array}{l}\text { Superior } \\
\text { Seedless }\end{array}$ & Média \\
\hline $12 / 2000$ & $0,17 \quad \mathrm{aB}$ & 0,07 & 0,30 & $0,08 \mathrm{bcC}$ & $0,04 \quad \mathrm{cC}$ & 0,13 \\
\hline $06 / 2001$ & $0,22 \mathrm{aB}$ & $0,19 \quad \mathrm{bBC}$ & 0,50 aA & 0,12 & 0,09 & 0,22 \\
\hline $10 / 2001$ & $0,14 \mathrm{aB}$ & 0,11 & $0,29 \quad \mathrm{bA}$ & 0,09 & 0,19 aAB & 0,16 \\
\hline $02 / 2002$ & $0,12 \mathrm{aB}$ & $0,05 \quad \mathrm{cB}$ & $0,25 \quad b A$ & $0,06 \quad \mathrm{cB}$ & 0,05 & 0,10 \\
\hline $04 / 2002$ & $0,19 \quad \mathrm{aB}$ & $0,26 \mathrm{aB}$ & 0,53 aA & 0,17 aB & $0,21 \quad \mathrm{aB}$ & 0,27 \\
\hline Média & 0,17 & 0,14 & 0,37 & 0,10 & 0,12 & \\
\hline C.V. $(\%)$ & 66,08 & 44,88 & 44,21 & 50,63 & 38,71 & \\
\hline
\end{tabular}

${ }^{1}$ Médias seguidas pela mesma letra minúscula na coluna e maiúscula na linha não diferem entre si pelo teste de Duncan a $1 \%$ de probabilidade 

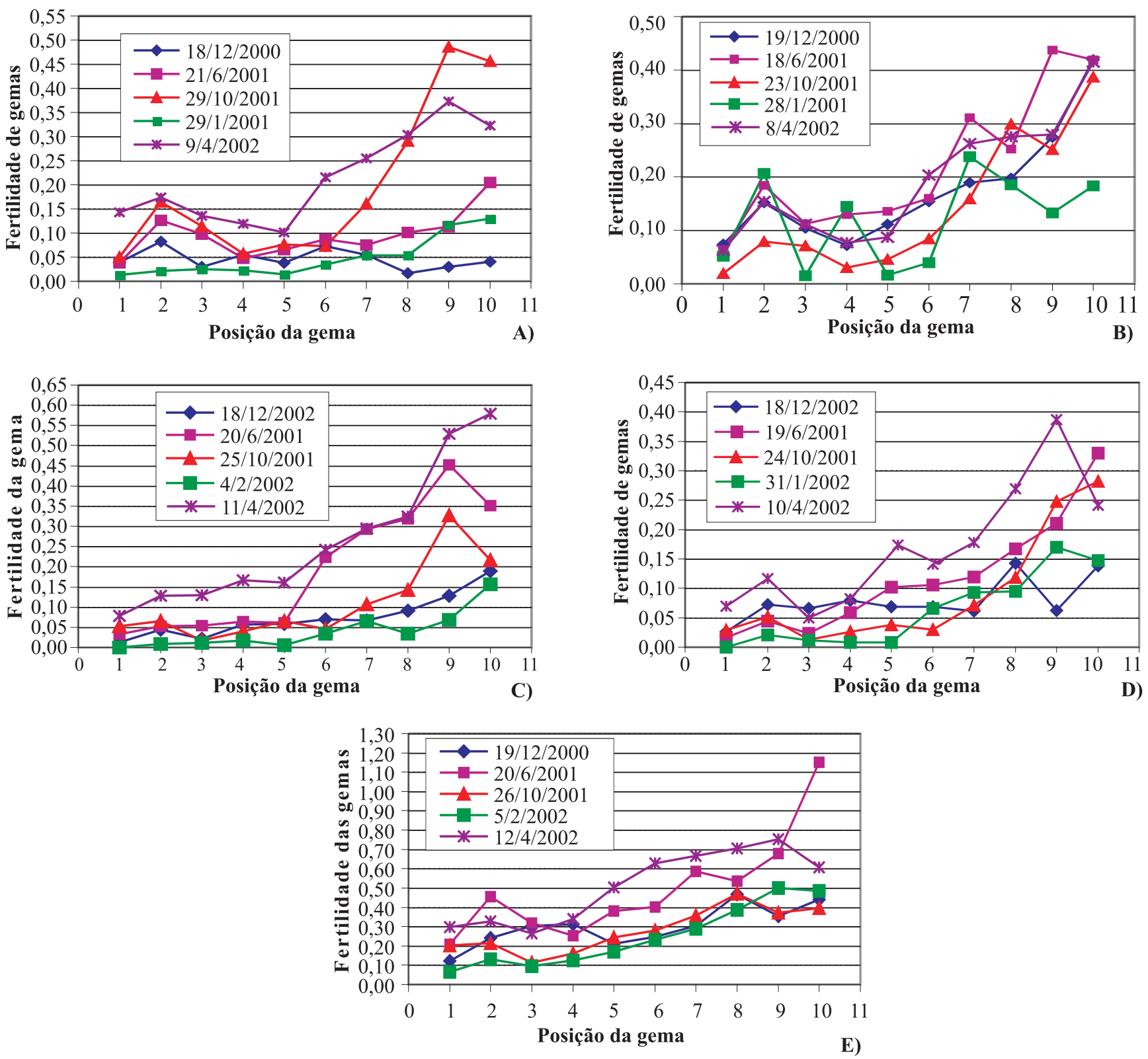

FIGURA 1 - Índice de fertilidade real de gemas de acordo com a sua posição na vara em cinco épocas de poda para as variedades de uvas sem sementes Superior Seedless (A), Perlette (B), Thompson Seedless (C), Catalunha (D) e Marroo Seedless (E).

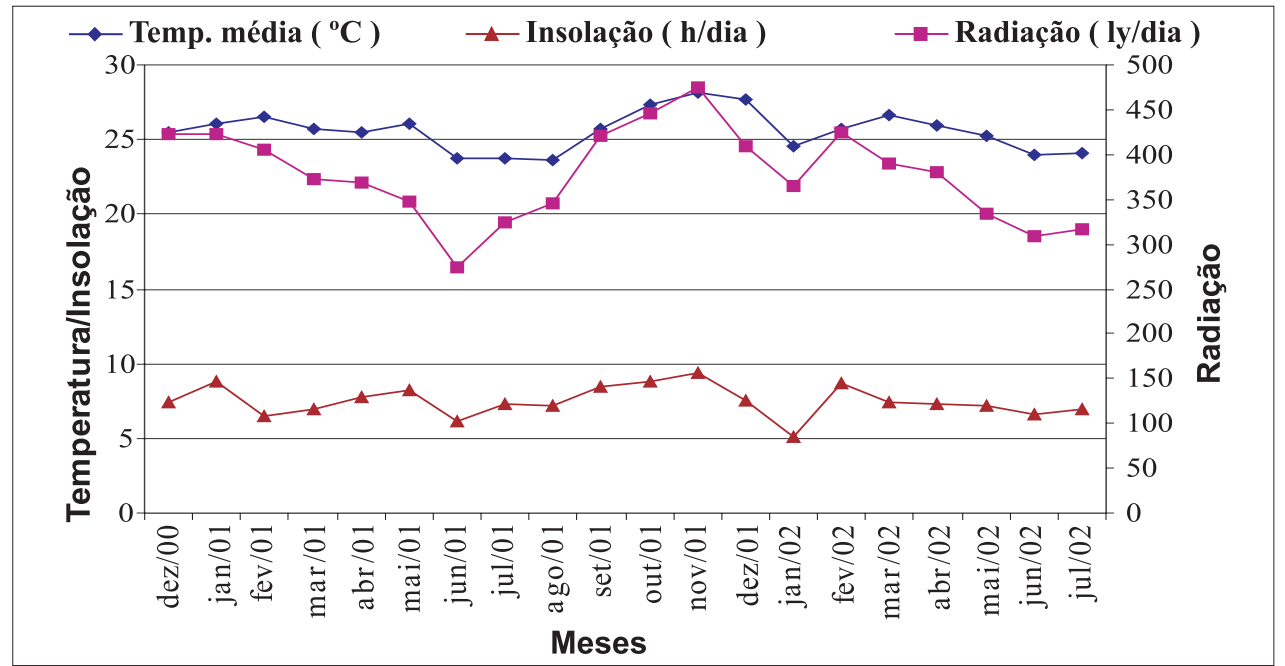

FIGURA 2 - Médias mensais de temperatura média $\left({ }^{\circ} \mathrm{C}\right)$, insolação (horas/dia) e radiação solar (ly/dia) de dezembro de 2000 a julho de 2002 no Campo Experimental de Bebedouro, Petrolina, PE. 
1). A fertilidade das gemas não apresentou diferenças significativas entre estas duas épocas de poda (06/2001 e 04/2002) nas variedades Perlette e Marroo Seedless. Índices de fertilidade de gemas mais baixos, por sua vez, foram obtidos quando a poda foi realizada em fevereiro de 2002 em todas as variedades. A média geral para os índices de fertilidade de gemas variou de 0,10 na poda de fevereiro de 2002 a 0,27 em abril de 2002.

As gemas, conforme pode ser observado na Figura 2, apresentam uma tendência de aumento de sua fertilidade a partir da porção mediana do ramo até a porção distal, apesar de poder ocorrer uma certa variação entre as gemas de posições adjacentes. As variações de fertilidade entre a gema basal e a $10^{\text {a }}$ gema da vara, considerando-se as cinco épocas de poda, foram, respectivamente, de 0,02-0,07 a 0,18-0,42 na 'Perlette'; 0,0-0,08 a 0,16-0,58 na 'Thompson Seedless'; 0,0-0,07 a 0,14-0,33 na 'Catalunha'; 0,06-0,30 a 0,40-1,15 na 'Marroo Seedless' e 0,01-0,14 a 0,04-0,46 na 'Superior Seedless'. Os valores médios de fertilidade de gemas obtidos para esta última variedade estão de acordo com aqueles observados por Sansavini \& Fanigliulo (1998), na Itália, variando de 0,03 a 0,6. A grande variação existente para esta variável é confirmada pelos valores altos de coeficientes de variação obtidos (Tabela 2).

A tendência de aumento da fertilidade a partir da porção mediana das varas é conseqüência de um maior acúmulo de carboidratos nesta região (Winkler, 1965). A baixa fertilidade das gemas basais também pode ser influenciada pela menor incidência de luz sobre essas gemas (May, 1966; May et al., 1976).

As épocas de poda no $1^{\circ}$ semestre do ano que ocorreu em junho de 2001 e abril de 2002, parecem favorecer tanto a brotação quanto a fertilidade de gemas da maioria das variedades estudadas. Isso se justifica pelo fato de que o ciclo anterior a estes iniciou em novembro de 2001 e dezembro de 2000 , cujo ciclo vegetativo coincidiu com período de temperaturas mais elevadas (Figura 1), o que pode ter favorecido a diferenciação das gemas.

\section{CONCLUSÕES}

1) A variedade Marroo Seedless destacou-se entre as variedades estudadas, com índice de fertilidade de gemas de 0,37, para os cinco ciclos de produção estudados;

2) O comportamento das variedades foi influenciado pelas épocas de poda, sendo que para a maioria das variedades as podas realizadas em junho de 2001 e abril de 2002 proporcionaram os maiores valores para brotação e fertilidade de gemas;

3) A fertilidade de gemas apresentou tendência crescente da base, representada pela $1^{\text {a }}$ gema, até a porção mediana que correspondeu a $10^{\mathrm{a}}$ gema das varas, e em geral as maiores médias foram observadas nas últimas gemas em todos as variedades.

4) A brotação e fertilidade das gemas pode sofrer variações em cada ciclo de acordo com as condições climáticas predominantes, sendo importante, a realização de análise de gemas antes da poda e de práticas adequadas de manejo de copa específicas para cada variedade.

\section{AGRADECIMENTOS}

Ao colega pesquisador da Embrapa Semi-Árido Clementino Marco B. de Faria pela valiosa colaboração na realização das análises estatísticas.

\section{REFERÊNCIAS BIBLIOGRÁFICAS}

BALDWIN, J.G. The relation between weather and fruitfulness of the Sultana vine. Australian Journal of Agricultural Research, East Melbourne, n.15, p.920-928, 1964.

BUTTROSE, M. S. Climatic factors and fruitfulness in grapevines. Horticultural Abstracts, Farnham Royal, v.44, n.6, p. 319-26, 1974.

BUTTROSE, M. S. Fruitfulness in grapevines:effects of changes in temperature and light regimes. Botanical Gazette, Chicago, n.130, p.173-179, 1969.

BUTTROSE, M. S. Fruitfulness in grapevines: the response of different cultivars to light, temperature and day lenght. Vitis, Geneva, NY, v.9, p.121-125, 1970 .

CAMARGO, U. A., CZERMAINSKI, A. B. C., MASHIMA, C. H. Fertilidade de gemas cultivadas de uvas apirênicas no Vale do Submédio São Francisco. In: CONGRESSO BRASILEIRO DE VITICUlTURAE ENOLOGIA, 8., 1996, Bento Gonçalves. Programa e Resumos... Bento Gonçalves: EMBRAPA-CNPUV, 1996. p.70.

LAVEE, S.; REGEV, U.; SAMISH, R. M. The determination of induction anddifferentiation in grapevines, Vitis, Geneva, NY, n.6, p.1-13, 1967.

LEÃO, P. C. de S.; PEREIRA, F. M. Estudo da brotação e da fertilidade das gemas de cultivares de uvas sem sementes nas condições tropicais do Vale do Submédio São Francisco. Revista Brasileira de Fruticultura, Jaboticabal, v.23, n.1, p.30-34, 2001.

MAY, P.; ANTCLIFF, A J. The effect of shadding on fruitfulness and yield in the Sultana. Journal of Horticultural Science, Ashford, n.38, p.85-94, 1963.

MAY, P. The effect of direction of shoot growth on fruitfulness and yield of Sultana vines. Australian Journal Agricultural Research, East Melbourne, v.17, p.491-502, 1966.

MAY, P.; CLINGELIFFER, P. R.; BRIEN, C. J. Sultana (Vitis vinifera L.) canes and their exposure to light. Vitis, Geneva, NY, v.14, p.278288, 1976.

MURRIEDA, A. L. Effecto de la longitud de poda en la fructificacion de vid Vitis vinifera L., cultivares Thompson Seedless y Málaga Roja. Agricultura Tecnica en Mexico, v.12, n.1, p.39-51,1986.

RIVES, M. Vigour, pruning cropping in the grapevine (Vitis vinifera L.). I. A literature review.

Agronomie, Paris, n.20, p.79-91, 2000.

SANSAVINI, S., FANIGLIULO, G. Fertilità delle gemme e influenza della potatura sullafruttificazione delle uve apirene "Centennial Seedless" e "Sugraone". Rivista di Frutticoltura, n.2, p.55-60, 1998.

SOMMER, K. J.; ISLAM, M. T.; CLINGELEFFER, P. R. Light and temperature effects on shoot fruitfulness in Vitis vinifera L. cv. Sultana: influence of trellis type and grafting. Australian Journal of Grape and Wine Research, n.6, p.99-108, 2000.

SRINIVASAN, C., MULLINS, M.G. Physiology of flowering in the grapevine - a review. American Journal of Enology and Viticulture, Reedley, v.32, p.47-63, 1981.

TONIETTO, J., CZERMAINSKI, A. B. C. Brotação e fertilidade das gemas da videira 'Cabernet Franc'. Revista Brasileira de Fruticultura, Cruz das Almas, v.15, n.1, p.185-192, 1993.

VALOR, O., BAUTISTA, D. Brotacion y fertilidad de yemas en tres cultivares de vid para vino. Agronomia Tropical, Maracay, v.47, n.3, p.347-58, 1997.

WINKLER, A. J. Viticultura. México:Continental, 1965. 792p. 